\title{
Database of Ground Level Enhancements (GLE) of high energy solar proton events
}

\author{
Ilya G. Usoskin* \\ Sodankylä Geophysical Observatory and ReSoLVE Center of Excellence, University of Oulu, \\ Finland \\ E-mail: ilya.usoskindoulu.fi
}

\section{Askar Ibragimov}

University of Helsinki, Finland

\section{Margaret A. Shea}

SSSRC, 100 Tennyson Avenue, Nashua, NH 03062, USA

\section{Don F. Smart}

SSSRC, 100 Tennyson Avenue, Nashua, NH 03062, USA

\begin{abstract}
Solar proton events (SPE) occur as a result of massive acceleration of charged particles in the solar corona and/or interplanetary space. Usually such events provide quite a soft spectrum of energetic particles, but sometimes the spectrum is sufficiently hard that the initial solar protons can generate secondary nucleons that can be detected as an increase in the cosmic ray flux at ground level. Such exceptional events are called GLE (ground-level enhancements) of cosmic rays and are numbered consecutively from the first event that was detected in 1942. Here we present an international GLE database hosted by the University of Oulu, that is an inheritance of pervious similar databases developed in the USA and Australia. We acknowledge the work of Louise Gentile, Mark Duldig and Harm Moraal.

The database is located at http://gle.oulu.fi

The interface and data organization are discussed as well as a possibility for accessing and mirroring the database.
\end{abstract}

The 34th International Cosmic Ray Conference,

30 July- 6 August, 2015

The Hague, The Netherlands

\footnotetext{
*Speaker.
} 


\section{Introduction}

Solar proton events (SPE) occur as a result of eruptive phenomena on the Sun that lead to massive acceleration of charged particles in the solar corona and/or interplanetary space [U, []]. Such events occur often around the solar cycle maximum and at the declining phase of solar cycles but they typically produce enhanced flux of particles with energy below a few hundred MeV. While such fluxes pose hazards for hardware and even human beings in space [3] they are not observed on the ground because of the shielding provided by Earth's atmosphere and at lower latitudes by Earth's magnetic field. However, sometimes SPEs may have a hard spectrum with the energy of solar energetic particles reaching several GeV. This energy is sufficient for the solar protons to initiate an atmospheric cascade generating secondaries that can reach the ground and be recorded by the ground-based cosmic ray detectors such as neutron monitors. Such exceptional events are called GLE (ground-level enhancements) of cosmic rays and occur, on average, roughly ten times per solar cycle. The occurrence of such events at Earth is related not only to the acceleration mechanism but also to the relative Sun-Earth geometry and the location of the parent acceleration region. GLE events are numbered consecutively from the first event that was detected in 1942. The most recent approved GLE event was \#71 in May 2012. According to convention, a SEP (solar energetic particle) event is counted as a GLE if at least two independent ground-based neutron monitors (NMs) recorded a simultaneous increase related to the arrival of the solar energetic particles as confirmed also by the satellite data if available. Since GLEs are measured on the ground, they are monitored since the introduction of neutron monitors in 1951 (before that time data from ionization chambers were used), thus providing information on solar energetic particles before the space era. Even in the space era, the GLEs remained the only source of data for the high energy portion of the SEP spectrum. Accordingly, it is important to collect verified data of GLEs that are accessible in one place.

The initial GLE database was developed in cooperation with the World Data Centers for the archiving and dissemination of these data [⿴囗十, 回]. The database was developed by M. A. Shea and D. F. Smart and maintained by Louise Gentile in the USA [ 6,0$]$. Later it was moved to Australia under the supervision of Marc Duldig. Finally, this International GLE database has reappeared at the University of Oulu. Additional datasets of GLEs have been developed by the North-West University (Potchestroom campus) in South Africa and in the framework of the NMDB project (http://nmdb.eu).

\section{The database}

The GLE DB [http://gle.oulu.fi] is stored in MySQL (MariaDB implementation) instance on a dedicated server belonging to the University of Oulu. The initial data is imported from "original" text files (inherited from previous generations of the GLE database) that are separately available for direct download at http://gle.oulu.fi/txtarchive and serve as a single source of data. The web interface is written in javascript using modern AngularJS, D3 and highcharts libraries, that allow for a modern look and feel. It allows to query any GLE and any station and examine a fully interactive chart of a GLE, and save it for further use (e.g., in scientific papers) and print. Back-end is written on PHP. Text file conversion to database is written on $\mathrm{C}++$. 


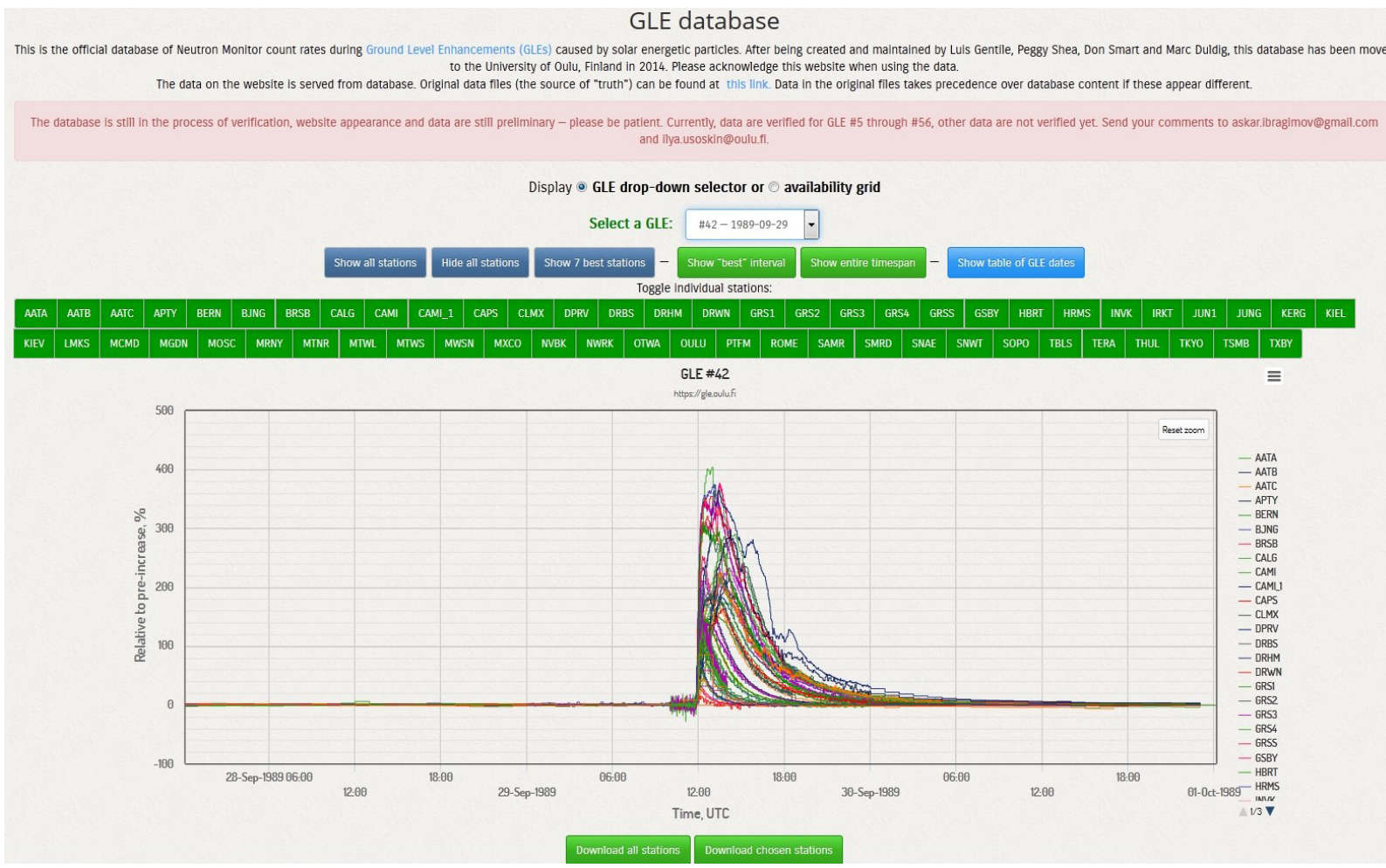

Figure 1: A screenshot of the GLE DB web-page (https://gle.oulu.fi).

A sample screenshot is shown in Fig. W for GLE \#42 of 30-Sep-1989. Below the basic header, information and links, there is a radio-button selection of the "GLE drop-down selection" or "availability grid". An example of the former selection is shown in Fig. 四, while the latter selection leads to another screen with a graphical representation of the station-event availability grid. A GLE event for analysis can be selected with the scroll-down menu below. The next row of buttons provides options for general adjustment of the plot - selecting or removing all stations and choosing the time interval. Below that one can find a number of green buttons with names (4-letter abbreviation) of individual NMs for which data are available for this particular event. Clicking on each button hides (the button becomes blue) or shows (green) the corresponding data on the main plot.

The main plot depicts temporal variability of count rates of all the available (or selected) NM stations for the analyzed event. The count rates are given in percent relative to the pre-increase level, which is defined in the original data files. The plot is interactive with a possibility to scroll and zoom the charts.

The green button below the main plot allows one to download, as a single zip-file, the ASCII text files in the standard GLE format with data for all (or selected) NM stations. An example of the data file in the standard GLE-format [5] is shown in Fig. ㅁ. Such files contain all the raw information so that a user can analyze real data, not only normalized percentage shown in the plot. The header contains basic information about the NM selected (name, location, type and number of counters, standard pressure and the barometric correction coefficient). The pre-increase base-line time interval is given, to which the percentage increase is normalized, as well as the scaling factors for different time resolution data. Next, the data are printed with the columns being: 


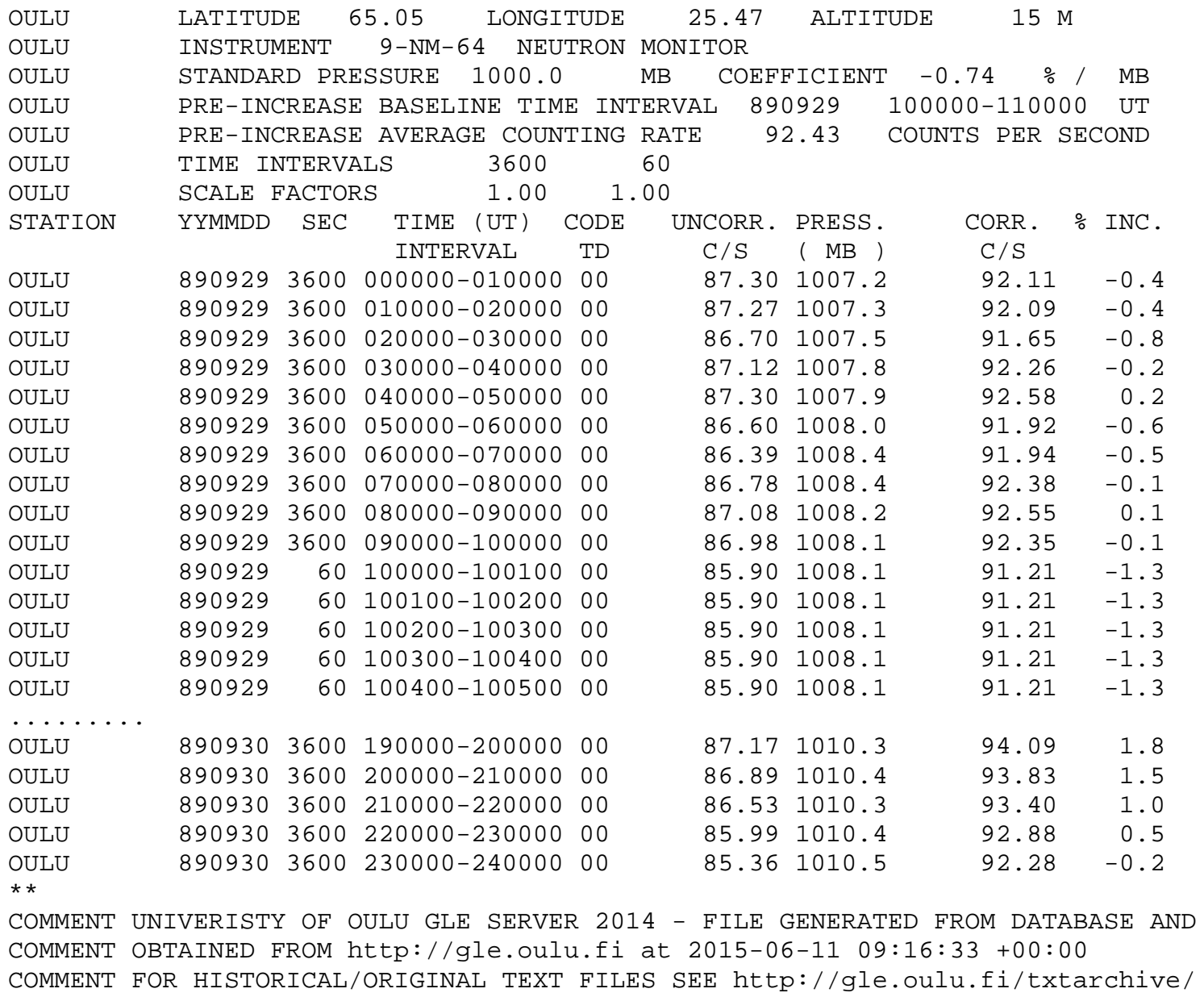

Figure 2: An example of the output ASCII text file with data (for Oulu NM for the GLE \#42) in GLE-format.

1. Station name;

2. Date in the format YYMMDD, first two digits of the year not shown, so that 89 means 1989 but 10 means 2010;

3. Time interval length in seconds;

4. Beginning and end of the time interval in the format HHMMSS;

5. Quality code;

6. Actual uncorrected count rate of the NM in counts per second;

7. Barometric pressure in units specified in the header (can be $\mathrm{mb}, \mathrm{hPa}$ or $\mathrm{mmHg}$ );

8. Corrected for pressure NM count rate;

9. Percentage increase with respect to the pre-increase baseline time interval. 
The last values in the last column are shown in the main plot. In the bottom of the file there is room for comments.

The original datasets contain data gaps and some apparent errors, especially in the earlier part of the time span. The work on verification and extension of the datasets is currently in progress, thanks to our assistants.

\section{Summary}

A new generation of the International GLE database is inaugurated at the web-site https://gle.oulu.fi

collecting and archiving data from the worldwide neutron monitor network concerning GLE events. The GLE database covers events starting from \#5 (23-Feb-1956) and provides a useful tool for an analysis of the most energetic solar particle events.

\section{Acknowledgements}

We acknowledge assistance of Teemu Willamo, Alexander Usoskin and Inna Usoskina in verifying the database records. IU's contribution was done in the framework of the ReSoLVE Centre of Excellence (Academy of Finland, project no. 272157).

\section{References}

[1] M. A. Shea and D. F. Smart, Fifty Years of Cosmic Radiation Data, Space Sci. Rev. 93 (2000) 229-262.

[2] D. F. Webb and T. A. Howard, Coronal Mass Ejections: Observations, Liv. Rev. Solar Phys. 9 (2012) 3.

[3] M. A. Shea and D. F. Smart, Space Weather and the Ground-Level Solar Proton Events of the 23rd Solar Cycle, Space Sci. Rev. 171 (2012) 161-188.

[4] M. A. Shea, D. F. Smart, M. Wada, and A. Inoue, A Suggested Standardized Format for Cosmic Ray Ground-Level Event Data, Proc. 19th Internat. Cosmic Ray Conf. 5 (1985) 510-513.

[5] M. A. Shea, D. F. Smart, J. E. Humble, E. O. Fluckiger, L. C. Gentile, J. E. Humble, M. Nichol, M. A. Shea, and D. F. Smart, A Revised Standard Format for Cosmic Ray Ground-Level Event Data, Proc. 20th Internat. Cosmic Ray Conf. 3 (1987) 171-174.

[6] L. C. Gentile, Relativistic solar proton database for the ground level enhancements during Solar Cycle 22, J. Geophys. res. 98 (1993) 21107.

[7] L. C. Gentile, J. M. Campbell, N. A. Vickers, S. M. Jacavanco, M. A. Shea, and D. F. Smart, $A$ Relativistic Solar Proton Database for the Ground-level Enhancements of 1956-1984, Proc. 23rd Inter. Cosmic Ray Conf. 3 (1993) 67-70. 